\title{
L'évaluation de la durabilité des fruitières à comté : vers un nouvel outil de management?
}

\author{
Philippe Jeanneaux
}

\section{OpenEdition}

Journals

Édition électronique

URL : http://journals.openedition.org/developpementdurable/8219

DOI : 10.4000/developpementdurable.8219

ISSN : 1772-9971

Éditeur

Association DD\&T

\section{Référence électronique}

Philippe Jeanneaux, «L'évaluation de la durabilité des fruitières à comté : vers un nouvel outil de management ? ", Développement durable et territoires [En ligne], Varia (2004-2010), mis en ligne le 17 septembre 2009, consulté le 04 mai 2019. URL : http://journals.openedition.org/

developpementdurable/8219; DOI : 10.4000/developpementdurable.8219

Ce document a été généré automatiquement le 4 mai 2019.

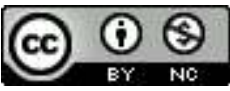

Développement Durable et Territoires est mis à disposition selon les termes de la licence Creative Commons Attribution - Pas d'Utilisation Commerciale 4.0 International. 


\title{
L'évaluation de la durabilité des fruitières à comté : vers un nouvel outil de management?
}

\author{
Philippe Jeanneaux
}

1 L'agriculture du massif jurassien est caractérisée par une orientation laitière importante, valorisée par la production de fromages sous signes officiels de qualité (cinq appellations d'origine contrôlée : comté, morbier, mont d'or, bleu de Gex, gruyère ; un label rouge : emmental grand cru). Le massif jurassien (limité ici au Doubs et au Jura) regroupait en 2004, plus de 3000 producteurs de lait à comté qui ont produit plus de 48000 tonnes de comté. Ce fromage a la particularité d'être produit par des petites unités de transformation: les fruitières. L'appellation d'origine contrôlée (AOC) comté a été le vecteur principal de l'organisation d'un système productif fromager original, fondé sur la division sociale et technique de la production. D'un côté, des éleveurs organisés en petits collectifs (150 fruitières en 2004) contrôlent la production de fromage en blanc (préaffiné), mais n'ont pas accès au marché. Ces fruitières à comté sont de petites entreprises à statut coopératif, gérées directement par les agriculteurs associés-coopérateurs. De l'autre côté, des entreprises d'affinage (une dizaine en 2004) ont le monopole de l'affinage et de l'accès au marché, mais n'investissent pas la première transformation. Cette forme de coopération économique a produit un surplus collectif durable, partagé équitablement, et sauvegardé par la construction réussie de l'appellation d'origine contrôlée comté (Perrier-Cornet, 1986) qui a notamment permis aux éleveurs d'avoir un prix du lait supérieur de $25 \%$ à la moyenne nationale au cours des dernières quinze années (traitement des données des services régionaux de l'information statistique et économique de Franche-Comté, 2008).

2 Mais cette organisation collective de la production est perturbée. Dans le contexte actuel très concurrentiel, la filière $\mathrm{AOC}$ comté connaît une restructuration importante. De grands groupes industriels prennent le contrôle des affineurs régionaux alors que les fruitières disparaissent, sans pour autant remettre en cause l'organisation de la filière comté (Jeanneaux, Perrier-Cornet, 1999). Plus de 70 fruitières ont disparu entre 1990 et 
2005. On en dénombre moins de 150 en 2009. Le plus souvent, elles se regroupent avec des coopératives voisines afin de répondre à la concurrence par la mise en œuvre d'une stratégie de domination par les coûts de production. D'autres fruitières ont développé leur avantage concurrentiel par la différenciation et/ou la diversification de leurs activités (Jeanneaux, Callois, Wouts, 2009).

Les fruitières-coopératives du massif jurassien sont donc pour certaines en difficulté. C'est pour cette raison qu'à la demande de la Fédération Départementale des Coopératives Laitières du Doubs (FDCL 25), nous nous sommes interrogés sur les possibilités de mise en œuvre d'un outil de pilotage des fruitières, adapté au traitement de ces stratégies concurrentielles, intégrant notamment des critères liés à l'ancrage territorial et au respect de l'environnement qui semblent devenir des facteurs essentiels de la pérennité des coopératives.

4 Avec cette recherche, nous visons deux objectifs : le premier objectif concerne l'étude des composantes de la durabilité et leur adaptation à un objet d'analyse spécifique : la petite et moyenne entreprise (PME) fromagère en général et la fruitière en particulier. Pour ce faire, nous traitons un ensemble de données d'un échantillon représentatif des fruitières. Son analyse nous permet de construire la grille de notation nécessaire à la mise en place des scores qui nous permettent de situer les niveaux relatifs de la performance globale (correspondant aux trois dimensions de la durabilité) de la fruitière. Le deuxième objectif concerne la réflexion sur la construction d'un nouvel outil de management des fruitières. Cet outil basé sur la prise en compte de critères nouveaux de durabilité devrait apporter un regard différent sur la performance globale des fruitières. Le tableau de bord intégrant de nouveaux indicateurs de durabilité jusqu'à présent ignorés, devrait aider les dirigeants à piloter plus finement la stratégie de leur fruitière. Il pourrait en outre, être un outil d'aide au positionnement concurrentiel des fruitières qui s'interrogent sur la nature de l'avantage concurrentiel à privilégier : domination par les coûts vs différenciation. Cette approche pourrait alors leur permettre d'identifier les leviers d'action sur lesquels agir pour assurer leur pérennité.

Pour atteindre ces deux objectifs, cet article est organisé en quatre points. Tout d'abord, nous préciserons notre démarche méthodologique (1.). Nous présenterons ensuite nos résultats sur la base des trois échelles de durabilité (2.). Nous discuterons ces résultats pour mettre en évidence trois catégories de fruitières identifiées selon leur niveau de durabilité et l'intérêt d'intégrer dans le management de la fruitière les objectifs du développement durable (3.).

\section{Le cadre méthodologique : évaluer la durabilité de la fruitière par une méthode de notation}

\subsection{Du cadre conceptuel de la durabilité aux objectifs de la méthode}

La mise en œuvre d'une méthode d'évaluation de la durabilité des fruitières du massif jurassien passe par la traduction concrète du principe de durabilité énoncé par le rapport Bruntland (WCED, 1987). La durabilité a été définie comme un mode de développement ayant pour but la satisfaction des besoins du présent sans compromettre la capacité des générations futures à satisfaire les leurs. A partir de ce principe, nous avons identifié les 
objectifs de durabilité correspondant aux fruitières du massif jurassien. Cette étape a permis d'énoncer nos hypothèses. Elle doit également servir à les discuter. Conformément à la définition de la durabilité, notre méthode d'évaluation est fondée sur les trois échelles de durabilité : sociale, environnementale et économique. Pour chaque échelle nous avons identifié les objectifs «opérationnels » qui pourront ensuite être évalués par une batterie d'indicateurs.

7 Notre démarche s'inspire des méthodes actuelles d'évaluation de la durabilité des exploitations agricoles (méthode IDEA (Vilain, 2003) (Zahm, Viaux, Girardin, Vilain, Mouchet, 2006 ), méthode du RAD (Réseau Agriculture Durable, CIVAM., 2001) et de leur adaptation aux spécificités des entreprises agricoles (Del'Homme, Pradel, 2005). Notre démarche s'appuie dans sa conception sur quatorze objectifs que nous avons regroupés selon les trois échelles de durabilité en tenant compte des spécificités des fruitières. Une coopérative fruitière est une structure collective, conçue pour être un outil au service de ses adhérents. L'idéal coopératif, c'est « donner aux agriculteurs la possibilité de maittriser leur propre destin et de promouvoir les progrès économiques et sociaux sur une base de solidarité et d'égalité » (Lasserre, 1977). Nous avons tenté d'intégrer dans la méthode d'évaluation les principes fondamentaux de la coopération qui sont les suivants : 1) La libre adhésion: c'est le principe de la «porte ouverte». Tous les producteurs dont l'activité entre dans l'objet social et qui demeurent à l'intérieur de la circonscription territoriale de la coopérative peuvent devenir adhérents sous réserve de l'approbation des autres sociétaires représentés par le conseil d'administration. 2) La double qualité : nul ne peut bénéficier des services de la coopérative sans en être membre, et réciproquement, nul ne peut être membre de la coopérative sans bénéficier de ses services. 3) La gestion démocratique : c'est le principe égalitaire : « un homme = une voix ». Il doit être appliqué dans toutes les assemblées générales à tous les sociétaires, quelle que soit l'importance de leur participation au capital et de leur activité économique avec la coopérative.

Les coopératives sont des sociétés de personnes et non pas des sociétés de capitaux dont les actionnaires peuvent à tout moment vendre leurs actions. La coopérative comme société de personnes véhiculent quatre valeurs essentielles : la transparence (les règles de fonctionnement de la coopérative doivent être claires et connues de tous); l'équité (le service rendu est le même pour tous, à qualité égale. Toutefois, une différenciation des adhérents permettant à chaque agriculteur de retrouver dans la coopérative le résultat de son engagement et de sa propre performance est admise. Les différences occultes et non justifiées économiquement sont prohibées); la solidarité (elle joue au bénéfice des sociétaires temporairement en difficulté) ; et la responsabilité (l'objectif de la coopérative est de permettre à chacun de ses membres de réussir pleinement en lui donnant, au-delà du seul intérêt économique, les moyens de comprendre son environnement pour mieux s'adapter).

9 Par ailleurs, la coopérative valorise deux options : 1) l'acapitalisme : ce terme signifie que la coopérative ne distribue jamais de dividendes et que les réserves sont impartageables entre les sociétaires. Les excédents réalisés sont versés à chacun, proportionnellement aux opérations qu'il effectue avec la société, sous la forme de ristournes. Il n'y a donc pas de bénéfices ou de dividendes au sens propre du terme. 2) Le développement de l'éducation: Toutes les sociétés coopératives doivent favoriser la formation aux principes de la coopération pour leurs membres, leurs dirigeants, leurs employés et pour le grand public. Nous avons alors décliné selon les trois échelles les principes de durabilité et de la coopération agricole. L'échelle sociale a été transformée en échelle socio-territoriale pour 
tenir compte de l'inscription des fruitières dans leur territoire. Elle prend en compte également les principes de gouvernance et de respect du fonctionnement de la démocratie, telles qu'ils sont promus dans l'idéal coopératif. L'échelle socio-territoriale regroupe les objectifs de : relation à la collectivité (la durabilité d'une collectivité humaine passe par l'implication de ses membres dans la vie sociale et du territoire. Cette implication permet le partage des risques et la résilience des structures. Le statut coopératif des fruitières contient en principe ces valeurs de solidarité et de mutualisation des risques); développement humain (l'épanouissement des individus dans leur milieu (notamment les salariés) est un facteur favorable à leur attachement et à leur stabilité dans le territoire); développement local (une fruitière s'inscrivant dans un système durable doit concourir au dynamisme économique et social du territoire) ; emploi (le maintien et la création d'emploi par les fruitières est un enjeu du développement durable); éthique (il n'est pas viable de ne considérer que les impératifs économiques individuels dans les choix de gestion. Les valeurs du respect de la vie et du commerce équitable (qualité des produits, prix permettant de rémunérer tous les maillons d'une filière...) sont à prendre en compte dans un système durable. Le statut coopératif contient également ces valeurs); qualité de vie (c'est un objectif du développement durable qui place la collectivité et les personnes humaines au centre de la démarche de développement) ; qualité des produits (la recherche de la qualité des produits traduit le respect du consommateur, permet le maintien des fruitières et donne une notoriété au territoire. Ce rôle dans l'aménagement du territoire va dans le sens d'un développement durable).

11 Nous n'avons intégré comme critère d'évaluation que ceux pouvant être discriminant entre les coopératives. Par conséquent, nous n'avons pas construit de critères concernant directement la question de l'équité car c'est une valeur consubstantielle des coopératives. En effet, le service rendu est le même pour tous, à qualité égale.

12 L'échelle environnementale. Elle concerne les objectifs de protection de l'atmosphère (c'est un objectif environnemental croissant en particulier avec le problème du réchauffement climatique et des gaz à effet de serre); de protection et gestion de l'eau (la gestion de la ressource en eau, en quantité et en qualité est une problématique clé du développement durable. Les coopératives fromagères sont d'importantes utilisatrices d'eau et sont également grandes productrices d'effluents); et de gestion économe des ressources naturelles non renouvelables (le développement durable encourage les pratiques qui prennent en compte les générations futures et leurs besoins. Il préconise donc la gestion prudente et économe des ressources).

13 L'échelle économique concerne les objectifs d'efficience (pour être durable l'entreprise doit limiter les gaspillages et bien valoriser ses productions pour assurer une rémunération correcte $\mathrm{du}$ prix $\mathrm{du}$ lait aux producteurs); de reproductibilité et transmissibilité de l'outil de production (la fruitière doit pouvoir être transmise aux générations futures ; c'est d'ailleurs un des principes forts du statut coopératif. L'outil de production est censé traverser les générations de producteurs. Son niveau de capitalisation ne doit être, ni trop faible, ni trop fort. Elle doit par conséquent, maîtriser son financement et contrôler son indépendance).

14 Nous leur avons ajouté deux objectifs transversaux. La dimension transversale permet de prendre en compte des objectifs valables pour les trois échelles : objectif d'adaptabilité (l'adaptation d'une fruitière à un environnement changeant est un facteur de sa pérennité) ; et d'objectif de cohérence (c'est un objectif général qui relie les préoccupations sociales, environnementales et économiques). 


\subsection{Des choix pour la construction des indicateurs et leur mode de calcul}

15 Pour évaluer la durabilité des fruitières et fournir un tableau de bord intégrant les trois échelles de la durabilité, nous nous sommes inspirés d'outils d'évaluation de la durabilité existants, basés sur la technique du scoring (méthode IDEA (Vilain, 2003), méthode du RAD (Réseau Agriculture Durable, CIVAM., 2001), méthode RISE (Häni, Braga, Stampfli, Keller, Fischer, Porsche, 2003)). Le scoring est une technique de notation qui consiste ici à attribuer des notes à des indicateurs de durabilité.

\section{Choix des indicateurs}

Tout d'abord, nous avons cherché à définir pour chaque objectif défini ci-dessus les indicateurs de durabilité correspondant. Par ailleurs, plusieurs indicateurs ont été sélectionnés à partir d'un travail d'analyse économétrique des déterminants de la pérennité des fruitières. Nous avions développé dans une précédente recherche (Jeanneauxet al., 2009), un modèle d'analyse discriminante sur des variables qualitatives traitant de la concentration horizontale des fruitières. Il avait été réalisé sur une base de données de 150 fruitières constituée à partir de l'exploitation du répertoire SIRENE, complétée par des informations collectées auprès des FDCL du Doubs et du Jura, pour les années 1990 et 2004. Quatre groupes de déterminants essentiels de la durabilité des fruitières avaient été repérés : (1) des déterminants économiques concernant la taille de la fruitière et le productivité des travailleurs, (2) des déterminants technologiques concernant l'adoption du ramassage du lait à la ferme et la mise aux normes de l'atelier de transformation, (3) des déterminants spatiaux concernant l'altitude et la localisation des fruitières les unes par rapport aux autres, et (4) des déterminants sociologiques concernant notamment le mode de gestion de la coopérative. Ensuite, nous avons ajouté des critères environnementaux et sociaux selon leur pertinence et leur capacité discriminante. Certains indicateurs ont été écartés tant ils étaient identiques pour toutes les fruitières. Il s'agit généralement de comportements de respect de la réglementation que toutes les coopératives adoptent ou des grands principes de la coopération comme l'équité par exemple. Enfin, certains indicateurs ont été éliminés car leur accès s'est révélé difficile. Dans d'autres cas, nous avons dû construire l'indicateur sur les bases d'une information qui fait l'objet d'une auto-évaluation par le président de la coopérative. Parmi les rubriques à remplir dans la grille, certaines sont renseignées par les FDCL du Doubs et du Jura et peuvent être entrées automatiquement à partir de la comptabilité. Par contre, 43 indicateurs (sur les 60 que compte notre méthode) doivent être renseignés par une enquête auprès des coopératives.

\section{La notation et la question de l'agrégation en une note unique globale}

Le principe de la méthode d'évaluation est de donner une note globale par fruitière pour chaque échelle de durabilité. Celles-ci ne seront jamais additionnées, l'addition de la somme des trois échelles n'a pas de sens. Chaque échelle est notée sur 100 points. Des totaux intermédiaires ont été calculés. Le nombre de points attribués à chaque indicateur est compris entre zéro et une valeur plafond qui est propre à chaque indicateur. L'ensemble des informations par échelle est décomposé en unités élémentaires de 
durabilité permettant ainsi de donner une note à chaque indicateur. Le score de chaque échelle de durabilité est le résultat de l'addition des notes de chaque unité élémentaire. Par conséquent, plus la note est élevée, plus la fruitière est censée avoir une durabilité élevée.

\section{La question de l'échelle des valeurs dans la notation}

Le problème principal est celui de retenir des échelles de valeur dans la notation qui tiennent compte de la contribution propre de chaque caractéristique retenue. De nombreuses questions de posent comme par exemple : pourquoi attribuer deux fois plus de poids à un critère qu'à un autre? Comment prendre en compte la non linéarité d'un facteur? Comment éviter la sur-représentation d'un critère ou les effets de dépendance à d'autres critères ? Peut-on agréger des notes au risque d'additionner des termes qui sont incompatibles entre eux? Doit-on accepter le principe de l'addition des indicateurs qui conduit à la compensation des points négatifs par des points positifs? En nous inspirant de la méthode du scoring, nous avons implicitement choisi de recourir à une méthode d'évaluation fondée sur des valeurs cardinales et moins sur des valeurs ordinales qui viseraient plutôt à opérer des classements entre critères et/ou entre individus. Nous sommes bien conscients que pour limiter ces biais, il conviendrait d'utiliser des outils plus élaborés et de renouveler les tests de sensibilité (Bockstaller, 2008).

Pour essayer de pallier cet ensemble de limites, nous avons établi les notations en fonction de différents critères. Tout d'abord, la pondération des notes a été retenue en fonction de la significativité des variables tirée des tests statistiques réalisés sur les données des coopératives pour les années 1990 et 2004, certains déterminants ayant plus d'effet que d'autres. Ensuite, la pondération a également pris en compte la répartition des réponses obtenues pour chaque indicateur en comparant le maximum, le minimum et la moyenne. La pondération a aussi pris en compte les appréciations des acteurs des fruitières (présidents de coopératives, FDCL, responsables de la profession agricole) avant la mesure de la durabilité des fruitières, puis après la présentation des résultats.

Enfin, cette démarche d'évaluation peut paraître statique. Elle est réalisée sur des données historiques, mais rien ne préjuge que les critères de durabilité que nous avons retenus ne demanderont pas des ajustements. Notre démarche n'est qu'une première étape qui ne fait que proposer une réflexion sur la mise en œuvre d'une démarche d'évaluation de la durabilité des fruitières et pose la question de l'aide multicritère à la décision à laquelle le décideur en action a à répondre. Elle permet de discuter du choix, $\mathrm{du}$ rangement, et du tri d'alternatives qui composent la décision. Plus qu'une note absolue obtenue pour chaque échelle, notre objectif vise à pouvoir relativiser des notes entre fruitières et à pointer les déterminants spécifiques de leur durabilité. Mais surtout, dans une deuxième étape, la démarche en permettant d'afficher explicitement de nouveaux indicateurs de performance, vise à ce que les associés-coopérateurs intègrent dans le management de leur fruitière la démarche dynamique l'amélioration continue de sa performance globale. 


\subsection{Organisation du contenu des trois échelles et détail de la composition des indicateurs}

\section{L'organisation générale des trois échelles d'évaluation}

21 La méthode est basée sur les trois dimensions prises en compte dans la définition du développement durable. Chaque dimension correspond à une échelle de durabilité. Les objectifs de l'échelle socio-territoriale se réfèrent davantage à l'éthique et au développement humain, caractéristiques essentielles des systèmes d'entreprise durables. L'échelle environnementale a pour objectif de permettre une bonne efficacité économique pour un coût écologique aussi faible que possible. L'échelle économique précise les notions essentielles liées à la fonction entrepreneuriale de la fruitière.

Chaque échelle est organisée en composantes, puis en critères intermédiaires, puis enfin en indicateurs. Une note est donnée pour chaque indicateur. Plus la note obtenue est élevée plus la fruitière est considérée comme durable. Pour chacune des trois échelles, la notation est réalisée à partir d'un système de points attribués à l'aide d'un abaque en fonction de la valeur de la variable considérée : l'échelle socio-territoriale est composée de 3 critères, 8 composantes et 24 indicateurs; L'échelle environnementale est composée de 4 critères, 9 composantes et 19 indicateurs; L'échelle économique est composée 4 critères, 7 composantes et 17 indicateurs. Nous présentons dans les tableaux ci-dessous $(1,2$ et 3$)$ les indicateurs et leur définition.

\section{Le détail de la composition des indicateurs}

23 L'échelle socio-territoriale. Elle se réfère à des valeurs sociales caractéristiques d'un certain niveau de socialisation qui sont implicites dans le développement durable (éthique, qualité, citoyenneté, développement humain). La fruitière durable crée de l'emploi, promeut un mode de gouvernance démocratique et participe au développement durable de son territoire en valorisant ses ressources spécifiques. L'ensemble des indicateurs et leur définition est précisé dans le tableau 1 ci-dessous. 
Tableau 1. Description des indicateurs de l'échelle socio-territoriale

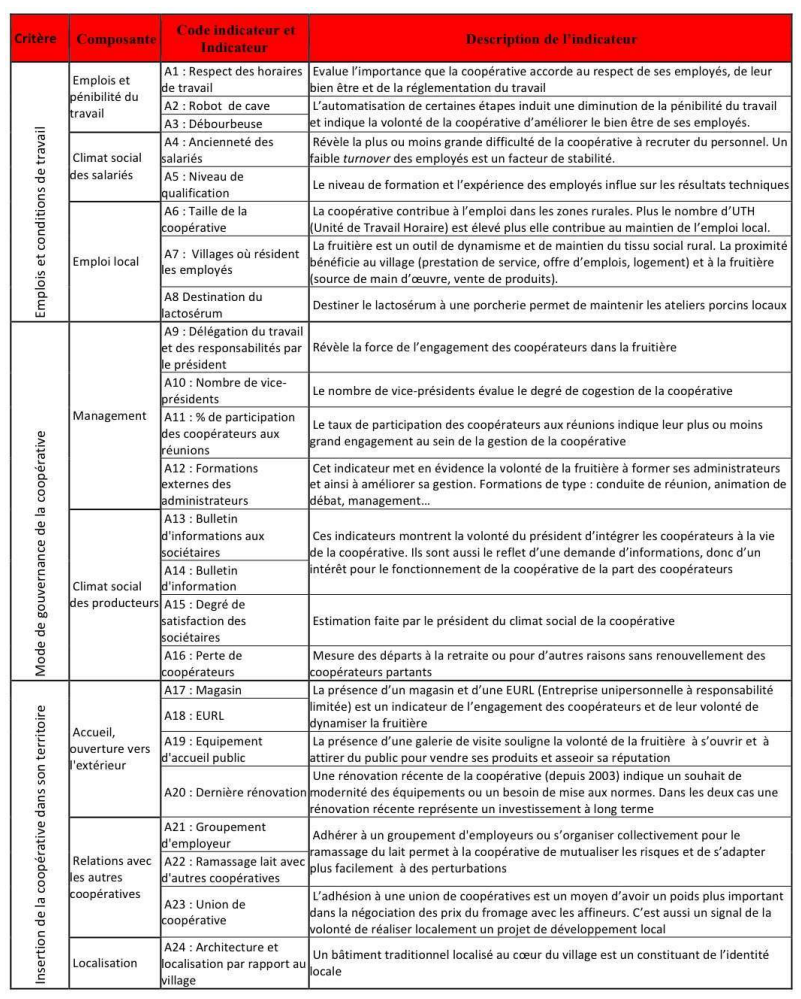

24 L'échelle environnementale. Elle se réfère à la préservation des ressources naturelles consommées par les entreprises (eau, air, paysage, énergies fossiles, matière premières, déchets). La fruitière durable consomme avec parcimonie ou utilise des énergies renouvelables. Elle limite ses pollutions et recycle ses déchets. L'ensemble des indicateurs et leur définition sont précisés dans le tableau 2. ci-dessous). 
Tableau 2. Description des indicateurs de l'échelle environnementale

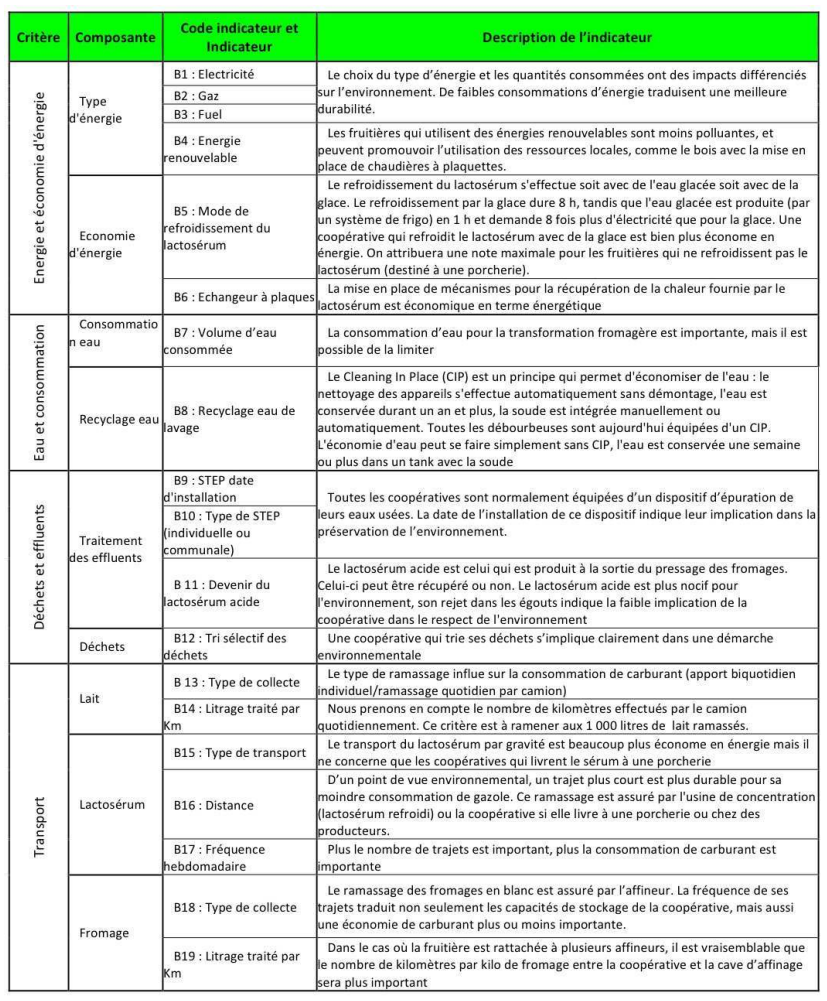

25 L'échelle économique. Elle précise les notions essentielles liées à la fonction entrepreneuriale de l'entreprise agroalimentaire et à la spécificité coopérative. La fruitière économiquement viable est transmissible, efficiente et indépendante financièrement. L'ensemble des indicateurs et leur définition est précisé dans le tableau 3. ci-dessous) 
Tableau 3. Description des indicateurs de l'échelle économique

\begin{tabular}{|c|c|c|c|}
\hline Critère & Composante & $\begin{array}{l}\text { Code indicateur et } \\
\text { Indicateur }\end{array}$ & Description de l'indicateur \\
\hline \multirow{4}{*}{ 芩 } & \multirow[t]{2}{*}{ Rendements } & $\begin{array}{l}\mathrm{C} 1 \text { : rendement matière } \\
\text { grasse }\end{array}$ & \multirow{2}{*}{$\begin{array}{l}\text { Plus le rendement en matière grasse et le rendement fromager sont élevés, plus la } \\
\text { coopérative est efficiente. }\end{array}$} \\
\hline & & $\mathrm{C} 2$ : rendement fromager & \\
\hline & \multirow{2}{*}{$\begin{array}{l}\text { Efficience } \\
\text { économique }\end{array}$} & \begin{tabular}{l|} 
C3 : Efficacité laitière \\
(CAF courante + prix du \\
lait par litre de lait)
\end{tabular} & $\begin{array}{l}\text { Traduit la capacité de la fruitière à maîtriser ses coûts de production, à payer un prix de } \\
\text { lait élevé et à conserver de la richesse pour reproduire l'outil }\end{array}$ \\
\hline & & $\begin{array}{l}\text { C4: Chiffre d'activités } \\
\text { par litre de lait }\end{array}$ & Traduit la capacité de la fruitière à produire en qualité et en quantité \\
\hline \multirow{7}{*}{ 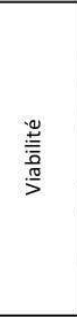 } & \multirow{4}{*}{$\begin{array}{l}\text { Viabilité } \\
\text { économique }\end{array}$} & C5 : Annuités/EBE & $\begin{array}{l}\text { Indique la capacité de la coopérative à faire face aux remboursements de ses dettes } \\
\text { aux banquiers }\end{array}$ \\
\hline & & C6: Prix du lait & Traduit la capacité de la coopérative à distribuer un prix de lait élevé \\
\hline & & $\begin{array}{l}\text { C7: Marge de } \\
\text { production }\end{array}$ & $\begin{array}{l}\text { Traduit la capacité de la coopérative à transformer plus de litrage que le seul litrage de } \\
\text { ses producteurs }\end{array}$ \\
\hline & & C8: Litrage & $\begin{array}{l}\text { Plus les quantité traitées sont importantes plus la coopérative réalise des économies } \\
\text { d'échelle et baisse ses coûts de production }\end{array}$ \\
\hline & \multirow{3}{*}{$\begin{array}{l}\text { Taux de } \\
\text { spécialisation }\end{array}$} & C9: comté & La production d'AOC comté est un moyen de bien valoriser le lait \\
\hline & & $\begin{array}{l}\text { C10: Pourcentage de } \\
\text { comté affiné }\end{array}$ & $\begin{array}{l}\text { Indique la valorisation commerciale du produit. La vente du fromage sur le lieu de } \\
\text { fabrication permet une meilleure valorisation grâce à l'effet « terroir ». }\end{array}$ \\
\hline & & C11: Autres fromages & $\begin{array}{l}\text { La capacité à produire d'autres fromages que le comté est un critère important } \\
\text { d'adaptation et notamment à transformer des laits hors « quotas » comté }\end{array}$ \\
\hline 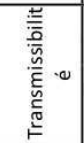 & $\begin{array}{l}\text { Appareil de } \\
\text { production }\end{array}$ & $\begin{array}{l}\text { C12 : Taux de vétusté et } \\
\text { Intensité capitalistique }\end{array}$ & L'outil de production est d'autant plus performant qu'il est récent \\
\hline \multirow{5}{*}{ 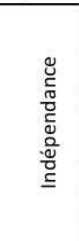 } & \multirow{3}{*}{$\begin{array}{l}\text { Indépendance } \\
\text { financière }\end{array}$} & \begin{tabular}{|l|} 
C13: Taux \\
d'endettement global
\end{tabular} & Indique la capacité de la coopérative contrôler le financement de l'outil de production \\
\hline & & $\begin{array}{l}\text { C14 : Fonds de } \\
\text { roulement }\end{array}$ & $\begin{array}{l}\text { Indique la capacité de la fruitière à accumuler de la richesse et à financer correctement } \\
\text { ses immobilisations avec des ressources financières permanentes }\end{array}$ \\
\hline & & $\begin{array}{l}\text { C15 : Solvabilité CT de la } \\
\text { coopérative }\end{array}$ & $\begin{array}{l}\text { Indique la capacité de la coopérative à faire face à des besoins de financement court } \\
\text { terme }\end{array}$ \\
\hline & \multirow{2}{*}{$\begin{array}{l}\text { Indépendance } \\
\text { sociale }\end{array}$} & C16: Nombre d'affineurs & Vendre à plusieurs affineurs permet de diluer les risques liés aux impayés. \\
\hline & & \begin{tabular}{|l|l} 
C17 : Devenir du \\
lactosérum
\end{tabular} & $\begin{array}{l}\text { La destination du lactosérum indique si la coopérative dispose de marge de manœuvre } \\
\text { en cas de défaillance d'un client }\end{array}$ \\
\hline
\end{tabular}

\subsection{Recueil et traitement des données}

Pour aboutir à la création d'un outil d'évaluation de la durabilité des fruitières, nous avons procédé par étapes. Quatre temps forts peuvent être identifiés, l'un correspondant au recueil de la donnée, l'autre à l'élaboration de la grille d'évaluation, puis au traitement des données et enfin à la présentation des résultats et aux ajustements de la méthodologie.

Une première phase de recherche documentaire a été réalisée pour mettre au point la grille d'évaluation. Elle a été complétée par une phase d'entretien avec les personnels (comptables, conseillers de gestion, directeur) de la FDCL du Doubs. Cette phase a permis de définir l'accessibilité à l'information nécessaire pour compléter notre grille d'évaluation. Nous avons ainsi pu définir les informations qui devaient faire l'objet d'un recueil spécifique auprès des fruitières. Nous avons décidé d'expérimenter la méthode et de limiter le recueil de données à 15 fruitières (25\% de l'effectif des fruitières du Doubs). Un tirage aléatoire de 15 fruitières réparties dans le département du Doubs a été réalisé. L'ensemble des fruitières de l'échantillon a été enquêté. Les données recueillies ont ensuite fait l'objet d'un traitement visant à attribuer des notes par indicateurs, puis par agrégation à donner une note globale par critère, puis par échelle. L'ensemble de l'information a été traitée sous Excel ${ }^{\oplus}$. Les résultats ont été présentés aux élus de la FDCL 25. Les remarques des professionnels ont permis d'ajuster la grille de notation. Il est toutefois ressorti que les élus restent encore peu sensibles aux critères environnementaux et sociaux contenus dans la méthode et restent très focalisés sur les indicateurs économiques et financiers. 


\section{Les résultats de l'évaluation de la durabilité de 15 fruitières du Doubs}

Nous avons regroupé les notes de toutes les fruitières dans une grille de notation afin de les comparer les unes aux autres. Pour chaque échelle, nous avons représenté la distribution des notes sur 100. Cela permet de mettre en évidence les coopératives plus ou moins durables selon nos critères d'évaluation, ainsi que la variabilité des résultats. Les notes globales par échelle sont les suivantes : pour l'échelle socio-territoriale, les notes varient entre 33 et 67 points sur 100 ; pour l'échelle environnementale, les notes varient entre 35 et 65 points sur 100 ; pour l'échelle économique, les notes varient entre 14 et 63 points sur 100. Parmi les 15 fruitières enquêtées, une fruitière se démarque des autres, en cumulant les meilleures notes sur les trois échelles.

\subsection{Analyse par échelle socio-territoriale}

Les notes s'échelonnent de 33 à 67 points pour un maximum possible de 100 points. Les notes moyennes attribuées pour chaque composante sont présentées dans la figure $1 \mathrm{ci}-$ dessous. Les résultats peuvent être ordonnés en deux blocs.

D'un côté, les coopératives qui obtiennent les meilleurs scores (plus de 60 points sur 100) sont des fruitières qui doivent leur note élevée à une combinaison de choix qui leur permettent d'assurer un bon climat social. Elles ont vraisemblablement su développer un projet social d'entreprise dans lequel les salariés bénéficient d'équipements modernes, qui limitent la pénibilité du travail, et qui permettent aux fromagers de réduire leur temps de travail tout en augmentant leur productivité. Sur le plan de la gouvernance, elles se démarquent également, parce qu'elles développent leur projet collectivement. La délégation et/ou la prise collective des décisions semblent être le mode privilégié de gestion des affaires de ces coopératives de taille supérieure ( 2 à 3 millions de litres de lait par an) ou qui sont engagées dans une stratégie de recherche d'un avantage concurrentiel par la différenciation. Il ressort enfin que les plus grosses fruitières ( 3 à 7 millions de litres de lait par an), peut-être parce qu'elles sont engagées dans des logiques de réduction des coûts de production plus affirmées, sont moins sensibles à leur insertion dans leur territoire. Elles ressentent peut-être moins le besoin de nouer des partenariats avec leurs voisines pour partager des salariés et des matériels.

31 A l'opposé, les coopératives qui obtiennent les moins bons scores (moins de 45 points) ont de très faibles capacités à produire de l'emploi et à assurer un projet professionnel motivant pour leurs travailleurs. Ces fruitières ont des tailles faibles (moins de 2 millions de litre de lait). Ce type de coopératives dégage insuffisamment de richesses pour investir dans des équipements de confort des fromagers. Elles ne sont pas en mesure de se moderniser et d'augmenter leurs gains de productivité, et se trouvent en position de repli. Une autre faiblesse est à noter au niveau de leur mode de gouvernance. Les coopérateurs sont moins impliqués dans la vie de leur coopérative et se comportent plus comme des fournisseurs de lait que comme des associés-coopérateurs apporteurs de lait AOC comté. Cette position se trouve dans certains cas exacerbée par un mode de gestion de la coopérative très individuel, par un président déléguant peu. 

dans leur territoire. Elles sont sans doute plus enclines à chercher des partenariats pour mutualiser des actifs afin de réduire les coûts ou pour mieux valoriser une partie de leurs produits par la vente directe.

Figure 1 : score moyen des 15 frutières pour l'échelle socio-temporelle

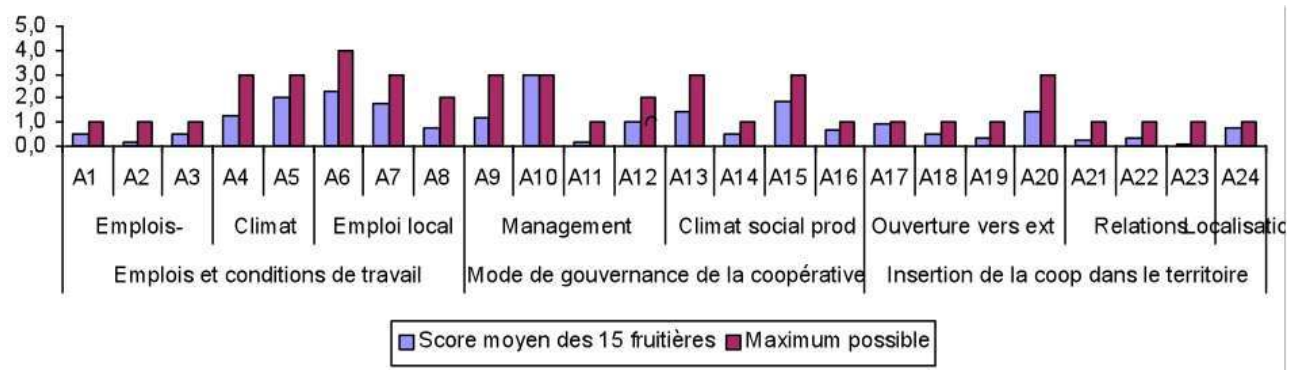

\subsection{Analyse par échelle environnementale}

Les notes s'échelonnent de 35 à 65 points pour un maximum possible de 100 points. Les notes moyennes attribuées pour chaque composante sont présentées dans la figure 2 cidessous.

Figure 2: score moyen des 15 fruitières pour l'échelle environnemental

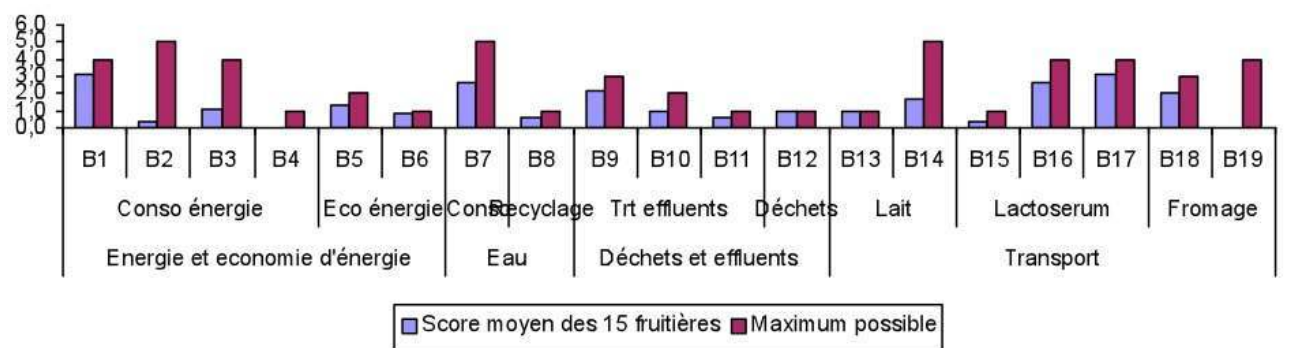

Neuf notes sur quinze sont inférieures ou égales à 50 points. Aucune coopérative de notre échantillon n'a opté pour les énergies renouvelables, ce qui pénalise l'ensemble des individus. Les scores les plus élevés sont obtenus par des coopératives qui cherchent à réduire leur consommation d'énergie, qui maîtrisent le devenir de leurs effluents et qui limitent leurs coûts de transport.

Tout d'abord, la réduction de la consommation d'énergie passe en priorité par des équipements permettant de récupérer la chaleur du lactosérum (échangeur à plaque, isolation de citerne...). Ensuite, quelques fruitières ne sont toujours pas raccordées à une station d'épuration et sont par conséquent fortement pénalisés. Enfin, les faibles coûts de transport sont obtenus lorsque la coopérative est située en zone de montagne, zone dont la densité laitière est plus forte. Les rares coopératives de la zone de plaine doivent couvrir un territoire beaucoup plus vaste pour collecter le lait. Les coopératives qui valorisent leur lactosérum en porcherie reçoivent également de bonnes notes. Cette solution de proximité présente l'avantage de limiter les coûts de transport et de déshydratation du lactosérum, mais fait parfois l'objet de polémiques, car elle est considérée comme une source de nuisances locales (odeurs et risques de pollution par le lisier). 


\subsection{Analyse par échelle économique}

\section{importante pour cette échelle. Les notes moyennes attribuées pour chaque composante} sont présentées dans la figure 3 ci-dessous.

Figure 3: score moyen des 15 fruitières pour l'échelle économique

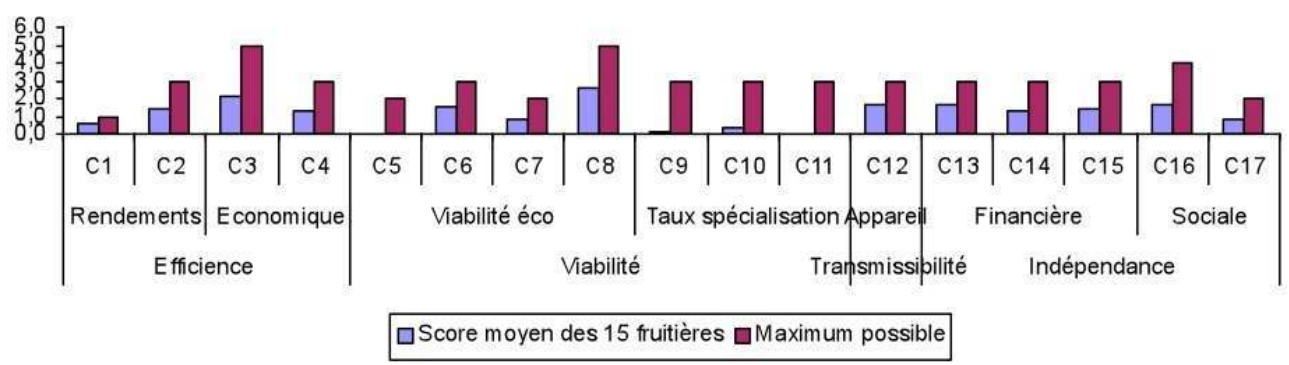

Les coopératives les plus durables sur le plan économique sont en priorité les fruitières de grandes tailles qui ont opté pour une stratégie de baisse des coûts. Toutefois, certaines grosses fruitières ont des scores faibles (moins de 40 points). Nous observons également que certaines petites structures ayant opté pour une stratégie affirmée de différenciation et de diversification (vente directe et production d'une deuxième, voire d'une troisième production fromagère : $\mathrm{AOC}$ mont d'or ou morbier) en continuant de produire du comté, ont les scores les plus élevés. Par contre, les petites coopératives en comté, sans diversification, ont une capacité à dégager de la richesse trop faible pour rémunérer le lait à un prix élevé, tout en conservant suffisamment de capitaux pour moderniser l'outil de production. Leur outil se détériore et/ou elles s'endettent. Devenues fortement dépendantes et difficilement transmissibles, elles semblent avoir un avenir compromis.

\section{Discussion : du diagnostic de la durabilité vers la mise en œuvre d'une démarche d'amélioration continue de la performance globale}

Cette analyse de la durabilité d'un échantillon de 15 fruitières en AOC comté du département du Doubs permet de mettre en évidence que les performances des fruitières ne reposent pas seulement sur les seuls critères économiques. Nous avons constaté que les dimensions de développement durable dans les processus de transformation de la valeur jouaient un rôle important. Dans le champ du management stratégique, nous savons que la mission de la stratégie est définie comme la création de la valeur dans un périmètre d'activité spécifique (Acquier, 2008). Par conséquent, en intégrant d'autres objectifs de performance (sociaux et environnementaux) dans la stratégie, certaines fruitières nous semblent avoir expérimenté de nouvelles dimensions qui font que les clients acceptent durablement de payer un prix supérieur aux coûts de production (Porter, 1985). Nous avons pu construire trois groupes de fruitières qui nous semblent mettre en évidence des logiques d'intégration différenciées des composantes du développement durable. A l'instar d'Acquier (2008), il nous parait possible de rattacher au sein de ces différentes logiques, des pratiques qui permettent de rendre compte de 
l'engagement des entreprises sur des problématiques sociétales. Il y a d'un côté des pratiques relevant des figures imposées et de l'autre celle relevant des figures libres (Acquier, 2008). Les figures imposées correspondent à des pratiques communes à l'ensemble des fruitières. Elles relèvent plus du mimétisme et de normes renforçant l'idée que la pérennité des fruitières est fondée sur des critères d'abord économiques et financiers. Les figures libres correspondent à des pratiques qui vont intégrer des dimensions innovantes qui vont au-delà de la réglementation et qui se singularisent par l'émergence d'actions renvoyant au développement durable. Les trois groupes de fruitières sont les suivants :

Tout d'abord, le groupe des grandes fruitières durables. L'analyse confirme que les coopératives de grandes tailles (ici entre 4 et 7 millions de litres de lait traités annuellement) ont les scores quasiment les plus élevés pour les trois échelles. Elles sont engagées dans des démarches collectives, démocratiques et de délégation de tâche et évitent ainsi de confier le management de la coopérative à une équipe administrative professionnelle. Leur forte capacité à dégager de la valeur ajoutée permet de bien rémunérer le lait aux producteurs, tout en finançant sans risque des investissements qui d'une part, améliorent le confort de travail des fromagers et en conséquence fidélisent les personnels, et les rendent plus productifs. D'autre part, mettent aux normes environnementales les outils. D'une certaine manière, les coopératives de ce premier groupe relèvent de pratiques dites « imposées ».

Mais, il ressort par ailleurs qu'une autre catégorie de coopératives est tout aussi durable. Il s'agit du groupe des coopératives durables «innovantes». Les fruitières de cette catégorie ont des tailles plus faibles et auraient plutôt des pratiques dites libres. N'ayant sans doute pas la possibilité d'augmenter leur taille, elles semblent avoir choisi d'inscrire leur avantage concurrentiel dans une logique affirmée de différenciation (voire de distinction) et/ou de diversification fromagère fondée sur un fort ancrage au territoire. Elles semblent avoir une très forte capacité à dégager de la richesse en valorisant les ressources spécifiques locales (main-d'œuvre, paysage, tradition, terroir, tourisme...). Les résultats de l'échelle socio-territoriale mettent en évidence cette caractéristique, ce qui jusqu'à présent n'avait pas été ressenti de manière aussi prégnante par les responsables des fruitières. Ayant des ressources financières pour investir dans leur outil, elles choisissent aussi de mettre aux normes sociales et environnementales leur outil.

41 Enfin, une troisième catégorie de fruitières faiblement durables ressort de l'analyse. Il s'agit de petites coopératives spécialisées en comté qui n'ont pas clairement choisi une des deux stratégies concurrentielles précédentes. Elles ne sont pas en mesure de réduire fortement leurs coûts de production, sans pour autant valoriser suffisamment leur ancrage territorial pour se différencier. Ayant de faibles capacités économiques, elles résistent en comprimant leurs dépenses d'investissement, notamment à dimensions sociales et environnementales. Elles semblent subir les évolutions du contexte économique et réglementaire et se trouvent fragilisées. Elles ont en quelque sorte opté pour des pratiques dites imposées alors qu'elles ont des caractéristiques (taille principalement) qui aurait dû les inciter à développer une stratégie de recherche d'un avantage concurrentiel hors coûts (différenciation ou diversification) plus affirmée.

42 Cette brève discussion montre l'intérêt qu'il y aurait à intégrer plus systématiquement dans le raisonnement managérial les critères de durabilité, notamment pour identifier les sources alternatives de performance. Elle met également en évidence la forte interdépendance des trois échelles de durabilité et le rôle de cette interdépendance sur la 
pérennité des fruitières. Autrement dit, avec cette première approche d'évaluation de la durabilité de fruitières, nous posons les bases d'une réflexion sur la responsabilité sociale (ou sociétale) des entreprises (RSE) (Rosé, 2006). Les fruitières ont en quelque sorte déjà intégré pour une grande majorité d'entre elles une meilleure prise en compte des impacts environnementaux et sociaux de leurs activités du fait peut-être de leur très fort ancrage territorial. Elles ne l'ont pas fait explicitement et spontanément. Elles ont répondu sans doute d'abord aux préoccupations de la société civile, mais aussi aux sollicitations de l'interprofession du comté qui depuis une quinzaine d'année a porté un projet motivant et innovant de développement local durable au sein du massif jurassien.

La question posée désormais est celle du pilotage stratégique de la performance globale au sein des fruitières. En effet, les fruitières confrontées au mouvement ancien et continu de concentration horizontale ont à s'interroger sur la définition, la mise en œuvre et l'ajustement de leurs orientations stratégiques. Par conséquent, la principale difficulté n'est pas tant de construire un diagnostic de la durabilité des fruitières à un temps $t$ que de leur faire prendre conscience de la réflexion qu'elles ont à mener sur la mise en œuvre d'une démarche de management de la performance globale, par définition dynamique et cognitive (Chabin, 2008). La caractérisation de la durabilité que nous avons réalisée pose les premières bases d'une démarche de conception d'un ensemble d'indicateurs de pilotage de la stratégie qui précisément est fondée sur une approche cognitive de la performance à l'instar des travaux développés par Chabin (2008). L'approche cognitive de la performance globale renvoie à l'idée d'une vision collective de la stratégie de la fruitière nourrie des contributions individuelles des acteurs de la fruitière et de ses parties prenantes. Pour ce faire, Chabin (2008, page 2) propose de recourir au tableau de bord stratégique et prospectif (TBSP) car il peut contribuer : au processus de construction de la stratégie et de traduction de ses objectifs au plan opérationnel. Le tableau de bord peut alors être construit avec l'ambition d'être multidimensionnel en intégrant des critères financiers et non financiers, de court et de long terme, qualitatifs et quantitatifs, rétrospectifs et perspectifs (Chabin, 2008), avec en toile de fond les indicateurs de durabilité proposés.

En définitive l'enjeu du tableau de bord est de poser les bases d'une démarche de management stratégique qui regroupe la phase de conception de la stratégie, celle de la construction de l'outil de pilotage et la phase de mise en œuvre. Il s'agit de disposer de moyen qui permettent d'identifier la contribution des actifs de la fruitière à la formation de la valeur et plus globalement à l'amélioration continue de la performance globale (Chabin, 2008).

\section{Conclusion}

45 Nous avons testé une grille d'évaluation de la durabilité des fruitières du Doubs qui s'appuie sur la technique du scoring. L'intérêt de cette méthode d'évaluation est de ne pas seulement prendre en compte des critères économiques, mais également des critères environnementaux et socio-territoriaux.

Notre démarche doit être considérée comme un support de réflexion pour les fruitières. Le tableau de bord qui devrait découler à terme de cette approche de la durabilité n'en est qu'au stade de la réflexion. Il n'a pas encore été formellement adopté par les coopératives. Elles nous semblent encore réticentes pour intégrer explicitement de nouveaux indicateurs sociaux et environnementaux dans leur management. Cette 
intégration de nouveaux indicateurs pourrait cependant permettre d'enrichir la réflexion stratégique des fruitières ainsi que la phase de contrôle des performances de la fruitière pour donner aux responsables des fruitières les moyens de piloter plus finement leur coopérative et d'améliorer de façon continue la performance globale de leur fruitière. Cet outil d'aide à la décision participe à la redéfinition du contenu des indicateurs de contrôle des performances utilisés dans le management des fruitières. Il peut permettre en outre, de pointer des pistes d'amélioration pour les fruitières identifiées comme peu durables, en mettant en évidence que le devenir de la fruitière ne repose pas uniquement sur une stratégie de domination par les coûts, mais aussi sur des stratégies de distinction fondées sur la valorisation des ressources spécifiques locales. Enfin, l'approche de la durabilité de la fruitière peut aider à sensibiliser les associés-coopérateurs à la notion de durabilité et de responsabilité sociétale des entreprises (RSE). Par conséquent, il nous semble que l'intégration dans le management des entreprises d'indicateurs de performance sociaux et environnementaux est un enjeu pour la pérennité des fruitières.

Enfin, sur le plan méthodologique, cette première démarche expérimentale contient de nombreuses limites qui sont de nature à limiter la portée de nos résultats. Des investigations sont envisagées pour améliorer les principes de notation des indicateurs et pour construire des tableaux de bord spécifiques à chaque fruitière.

\section{BIBLIOGRAPHIE}

Acquier A., 2008, "Développement durable et management stratégique : piloter un processus de transformation de la valeur", 17e Conférence Internationale de Management Stratégique Association Internationale de Management Stratégique (AIMS), Nice, 28-31 mai 2008, 27 p.

Bockstaller C., 2008, "La construction d'indicateurs", Ecole-Chercheurs "Démarches et méthodes pour l'évaluation multicritère de la durabilité des systèmes d'élevage et de culture", INRA, Tours, 22 mai 2008,

Chabin Y., 2008, "Pilotage et mesure de la performance stratégique en cave coopérative", Colloque SFER «les entreprises coopératives agricoles, mutations et perspectives, atelier E2, Paris, 28 et 29 février 2008, $11 \mathrm{p}$.

Del'Homme B., Pradel M., 2005, "Evaluation de la durabilité des exploitations viticoles dans le vignoble bordelais - Méthode et résultats", OENOMETRIE XII Macerata (Italie), 27-28 mai 2005, 23 p.

Häni F., Braga F., Stampfli A., Keller T., Fischer M., Porsche H., 2003, "Rise a tool for holistic sustainability assessment at the farm level", International Food and Agribusiness Management Review, vol. 6, n 4 , pp. 79-90.

Jeanneaux P., Callois J.-M., Wouts C., 2009, "Durabilité d'un compromis territorial dans un contexte de pression compétitive accrue : le cas de la filière AOC Comté", Revue d'économie régionale et urbaine, vol. 1, pp. 179-201 
Jeanneaux P., Perrier-Cornet P., 1999, "L'organisation en fruitière face à l'innovation technologique et au changement économique : Le cas de la filière comté", Colloque SFER-INRA-ENITA, Signes officiels de qualité et Développement Agricole - Aspect techniques et économiques, SFER, ENITAC, Clermont-Ferrand, les 14 et 15 avril 1999, 6 p.

Lasserre G., 1977, Les entreprises coopératives,Paris, PUF, Que sais-je., 127 p.

Perrier-Cornet P., 1986, "Le massif jurassien. Les paradoxes de la croissance en montagne. Eleveurs et marchands solidaires dans un système de rente", Cahiers d'Economie et de Sociologie Rurales, vol. $\mathrm{n}^{\circ} 2$, Avril 1986, pp. 62-121.

Porter M., 1985, Competitive Advantage: Creating and Sustaining Superior Performance,New York, The Free Press, 557 p.

Réseau Agriculture Durable, CIVAM., 2001, "Diagnostic de durabilité, Guide de l'utilisateur", in, http://perso.wanadoo.fr/agriculture-durable/francais/documents/guide.pdf, RAD et CIVAM, 8 pages

Rosé J.-J., 2006, Responsabilité sociale de l'entreprise. Pour un nouveau contrat social,Bruxelles, De Boeck, collection « Méthodes et Recherches ». 408 p.

Vilain L. (dir.), 2003, La méthode IDEA - Indicateurs de durabilité des exploitations agricoles, Dijon, Educagri éditions, $151 \mathrm{p}$.

WCED, 1987, Our Common Future (Brundtland-Report),(World Commission on Environment and Development), Oxford University Press, 437-495 p.

Zahm F., Viaux P., Girardin P., Vilain L., Mouchet C., 2006 "Farm Sustainability Assessment using the IDEA Method. From the concept of farm sustainability to case studies on French farms", Symposium INFASA, International Forum on Assessing Sustainability in Agriculture, IISD, Bern (Suisse), march 2006, 20 p.

\section{RÉSUMÉS}

Le massif jurassien est marqué par un système de production fromager basé sur la relation entre des petites coopératives de production de comté et des affineurs qui en assurent la finition et la commercialisation. Les fruitières sont fragilisées par la restructuration du secteur et s'interrogent sur leur devenir. Pour y répondre, nous avons développé une méthode d'évaluation de la durabilité des fruitières construite à partir de 60 indicateurs. Elle a été testée sur un échantillon de 15 fruitières. Nous montrons que leur situation ne s'explique pas par la seule prise en compte des performances économiques, mais également par la mesure de leurs caractéristiques socio-territoriales et environnementales. Nous posons l'idée que la pérennité des fruitières passera de plus en plus par la prise en compte dans leur démarche de management des principes du développement durable.

Sustainability assessment at the PDO comté cooperatives level: towards a new tool for management? The Jura region is based on a local organization between farming sector and industry. Numerous small cooperatives (the fruitières, which produce young cheese) are now threatened by the merger movement of the sector and they wonder about their future. We develop a method to assess the sustainability of these firms constructed around 60 indicators. We put to the test 15 cooperatives. We show their position depends not only on economical performances, but also on social, territorial and environmental features. We assume that the stake for the perenniality of the cooperatives is to take the sustainable principles into account in their management. 
INDEX

Mots-clés : coopérative-fruitière, filière AOC comté, management, indicateurs de durabilité

Keywords : Cooperative, PDO comté cluster, management, sustainability indicators

\section{AUTEUR}

\section{PHILIPPE JEANNEAUX}

Philippe Jeanneaux est Maître de conférences en économie rurale, ENITA Clermont - UMR Métafort 1273. Enita Clermont, BP 35, 63370 Lempdes (France) Tél. 33 (0)4 73987030.

jeanneaux@enitac.fr Dernières publications : Aznar O., Guérin M., Jeanneaux P., 2006, « La mise en oeuvre de politiques environnementales par des acteurs locaux. Étude sur une zone d'étude située en France ", Canadian Journal of Regional Science/Revue canadienne des sciences régionales, vol. XXIX, n 1, Spring/printemps 2006, p. 143-156. Jeanneaux P., Callois J.-M., Wouts C., 2009, "Durabilité d'un compromis territorial dans un contexte de pression compétitive accrue : le cas de la filière AOC Comté", Revue d'économie régionale et urbaine, vol. 1, pp. 179-201 Remerciements : Ce travail a été possible grâce à la contribution d'élèves ingénieurs en formation à l'Enita Clermont (Clémence Cros, Gracia de la Lastra et Elsa Oberlis) et au soutien financier de la Fédération Départementale des Coopératives Laitières du Doubs. 